\title{
Optimized FSO System Performance over Atmospheric Turbulence Channels with Pointing Error and Weather Conditions
}

\author{
Omar HASAN, Mohamed TAHA \\ Dept. of Communication Engineering, Princess Sumaya University for Technology, Amman, Jordan, 11941 \\ ohasan@psut.edu.jo,mtaha@psut.edu.jo \\ Manuscript received April 27, 2016
}

\begin{abstract}
In this paper, Bit Error rate and probability of outage are derived in closed form for plane wave mode over Gamma-Gamma free space optical channel under the influence of pointing errors and different weather conditions. The free-space optical system under study assumes deployment of intensity modulation / direct detection with on-off keying data formats. To evaluate the performance of the system using the derived expressions, two independent optimization algorithms were applied to find optimum system and channel parameters that optimize the performance of the two system metrics, i.e., bit error rate and probability of outage. The optimized system results were shown for different values of channel strength, weather conditions, optimum beamwidths and pointing error jitter variances. For each case, the optimized results are provided as a function of the transmitted power or average received power.
\end{abstract}

\section{Keywords}

Bit Error Rate (BER), Free Space Optical Channel (FSO), Intensity Modulation (IM), Direct Detection (DD) with On-Off Keying (OOK), pointing error, jitter variance

\section{Introduction}

Recent deployment of 4G technology resulted in great challenges to cellular carriers to increase backhaul capacity between cell towers. This increase in capacity is necessary to meet fast growing demands for mobile users acquiring deployment of $4 \mathrm{G}$ internet services. However, current installed microwave backhaul links cannot attain full $4 \mathrm{G}$ download speed for mobile users, hence, limiting number of users that can access the mobile network. On the other hand, optical cable technology can meet demands for higher speed data rates between backhaul cells, but its implementation can be difficult and expensive task due to different terrain types between backhaul cells. Free space optical (FSO) links can be a solution to meet growing demands by cellular carriers for higher link capacity as shown by [1]. It was shown by [1], FSO systems using ultrashort pulse (USP) lasers developed by [2], can provide $1 \mathrm{Gbits} / \mathrm{sec}$ and $10 \mathrm{Gbits} / \mathrm{sec}$ backhaul capacity for $2 \mathrm{~km}-$ $3 \mathrm{~km}$ backhaul distances in all weather conditions. In addition, FSO technology is low power, cost effective, no deployment licensing is required and can meet higher data rate capacity of future wireless technology such $5 \mathrm{G}$.

However, transmission of optical signals through FSO channels can be degraded due to atmospheric turbulence, pointing error and weather attenuations. Weather conditions such as rain, snow, haze and fog can highly degrade the optical signal and limit its coverage. In dense foggy weather conditions, an optical signal with operating wavelength $1550 \mathrm{~nm}$ can experience an attenuation of $270 \mathrm{~dB}$ per $\mathrm{km}$ [3] limiting link coverage distance to 200 meters. Atmospheric turbulence is caused by refractive index variations along the signal propagation path which results in signal fading (scintillation) at the receiver. Turbulence severity can be determined by the value of the Rytov variance. For Rytov variance $\ll 1.0$, turbulence channel is termed weak, as the Rytov variance exceeds unity value, turbulence strength varies from medium to strong until it reaches the saturation region at a Rytov value of 25 . Building sway causes misalignment (pointing error) between the transmitter and the receiver, therefore less optical power will be collected by the receiver which degrades the performance the FSO system.

Many authors have investigated the performance of FSO systems using different statistical models [4-16] to describe the channel randomness and its effect on the received signal. It was shown by [9], that the double-Weibull probability density function (pdf) is an appropriate model to describe the signal irradiance under medium to strong turbulence channel strengths. The log normal pdf was accurately used by [12], to study the signal irradiance fluctuation in weak turbulence conditions. It was shown by [11], that the gamma-gamma pdf is an appropriate and accurate channel model to describe the signal irradiance for weak to strong turbulence conditions. This pdf was then used and adopted by many authors [5-7], [12], [13], to evaluate the FSO system metrics performance for weak to strong turbulence conditions. 
This work was motivated by the ability of wireless FSO technology to provide high speed data rate for current and future wireless applications such as 4G/5G networks, medical technology, cloud computing and sensor networks. In the case of cellular network, FSO technology can overcome the challenges facing cellular carriers to provide higher backhaul capacity between cell towers as compared to current deployed microwave technology. Current FSO technology [2] is capable of providing maximum speed of $1 \mathrm{Gbit} / \mathrm{sec}$ between cell towers, resulting in an increase in the number of mobile users acquiring $4 \mathrm{G}$ and future $5 \mathrm{G}$ full speed internet connection. For example, a single backhaul microwave link can provide a maximum speed of $100 \mathrm{Mbit} / \mathrm{sec}$ between cell towers, limiting thereby the maximum number of mobile users that can access full $4 \mathrm{G}$ speed to one, hence, using 1 Gbit/sec FSO technology [2] can allow a maximum of 100 mobile users to access the full $4 \mathrm{G}$ internet speed. However, in order for the FSO system to provide such maximum backhaul capacity, FSO system needs to be optimized for the different system parameters, pointing error, channel and weather conditions. In this context, the derived expressions are used to optimize the beamwidths to achieve the maximum capacity under the previously mentioned conditions.

This work is different from previous published work [5], [6], [9], [10], [13], and [15], in the sense that the derived expressions are novel and closed form, and can provide insight into how to optimize the performance of the two system metrics, considering the combined effect of turbulence channel severity, pointing error, as well as the system parameters and weather conditions.

The rest of the paper is organized as follows. Section 2 shows analysis for the FSO system under study and derivation of the channel pdf which takes into account the combined effects of turbulence severity, weather conditions and pointing error. Closed form expressions for the probability of error and outage probability are derived in Sec. 3. This section also explains how the optimization routines can be implemented to optimize the performance of the two system metrics. In Sec. 4, optimized numerical results are presented for the two system metrics in terms of system and channel parameters, pointing error and weather conditions. Section 5 summarizes the work and provides comments and conclusions.

\section{System and Channel Models}

In this section, an FSO system employing intensity modulation / direct detection (IM/DD) with on-off keying (OOK) modulation is considered in the analysis below. The optical signal propagates through slowly fading gammagamma turbulence channel under the effect of pointing error, weather attenuation and additive white Gaussian noise. The received signal $y$ can be written as

$$
y=x \cdot R \cdot h+n
$$

where $x$ is the OOK modulated signal takes on the values 0 or 1 with average optical power $P_{\mathrm{t}}, R$ is the detector re- sponsivity and $n$ is an additive white Gaussian noise with variance $\sigma_{n}^{2}=N_{\mathrm{o}} / 2 \mathrm{~W} / \mathrm{Hz}$. The channel state $h$ is assumed to be the product of three independent factors [15]:

$$
h=h_{\mathrm{a}} \cdot h_{\mathrm{p}} \cdot h_{\mathrm{o}}
$$

the parameter $h_{\mathrm{a}}$ is random attenuation due to atmospheric turbulence, $h_{\mathrm{p}}$ is random attenuation due to geometric spread and pointing error, and $h_{\mathrm{o}}$ is constant laser power attenuation due to weather conditions which can be evaluated using Beers-Lambert law as [3]:

$$
h_{\mathrm{o}}(L)=\frac{P(L)}{P(0)}=\exp (-\sigma \cdot L)
$$

where $h_{\mathrm{o}}(L)$ is the transmittance at distance $L, P(L)$ is the laser power at distance $L$ from the source and $P(0)$ is the laser power at the source, and $\sigma$ is the attenuation coefficient and usually expressed in units of $\mathrm{dB} / \mathrm{km}$. The attenuation coefficient depends on the wavelength and the visibility range which can be evaluated using the empirical formula as in (6) from [3].

For OOK modulation the received electrical signal-tonoise ratio (SNR) can be represented as [15]

$$
u=\operatorname{SNR}(h)=\frac{2 P_{\mathrm{t}}^{2} \cdot R^{2} \cdot h^{2}}{\sigma_{\mathrm{n}}^{2}}
$$

by using (4), the average signal to noise ratio can be calculated as

$$
\bar{u}=E[\operatorname{SNR}(h)]=\frac{2 \cdot P_{\mathrm{t}}^{2} \cdot R^{2}}{\sigma_{\mathrm{n}}^{2}} \cdot E\left[h^{2}\right] .
$$

The notation $E[\cdot]$ denotes expectation and $h$ as in (2) is the combined channel state and will be derived in the following section.

\subsection{Atmospheric Turbulence Fading Model}

In this paper, gamma-gamma pdf model is considered to describe channel turbulence effects. In this case, the irradiance $h_{\mathrm{a}}$ can be expresses by the following distribution

$$
f_{h_{\mathrm{a}}}\left(h_{\mathrm{a}}\right)=\frac{2 \cdot(\alpha \beta)^{\frac{\alpha+\beta}{2}}}{\Gamma(\alpha) \Gamma(\beta)} h_{\mathrm{a}}^{\frac{\alpha+\beta}{2}-1} \cdot \mathrm{K}_{\alpha-\beta}\left(2 \sqrt{\alpha \beta h_{\mathrm{a}}}\right), h_{\mathrm{a}}>0
$$

where $\mathrm{K}_{v}(\cdot)$ is the $v$ th-order modified Bessel function of the second type, while $\Gamma(\cdot)$ is the gamma function, and the parameters $\alpha$ and $\beta$ represent the effective number of largescale and small scale cells of the scattering process given by $[11]$

$$
\alpha=\frac{1}{\sigma_{x}^{2}}, \beta=\frac{1}{\sigma_{y}^{2}} .
$$

The quantities $\sigma_{\mathrm{x}}^{2}$ and $\sigma_{\mathrm{y}}^{2}$ are the large scale and small scale scattering variances. Evaluation of the two variances $\sigma_{\mathrm{x}}{ }^{2}$ and $\sigma_{\mathrm{y}}{ }^{2}$ are directly related to the size of the inner scale 
scattering objects and the wave propagation mode. For plane wave and zero inner scale values, the variances $\sigma_{\mathrm{x}}^{2}$ and $\sigma_{\mathrm{y}}^{2}$ are evaluated using (18) and (19) from [11]

$$
\begin{aligned}
& \sigma_{x}^{2}=\exp \left[\frac{0.49 \cdot \sigma_{\mathrm{I}}^{2}}{\left(1+1.11 \cdot \sigma_{\mathrm{R}}^{12 / 5}\right)^{7 / 6}}\right]-1, \\
& \sigma_{\mathrm{y}}^{2}=\exp \left[\frac{0.51 \cdot \sigma_{\mathrm{I}}^{2}}{\left(1+0.69 \cdot \sigma_{\mathrm{R}}^{12 / 5}\right)^{5 / 6}}\right]-1 .
\end{aligned}
$$

The Rytov variance $\sigma_{\mathrm{R}}{ }^{2}$ is usually used to characterize the strength of optical scintillation given by [11]

$$
\sigma_{\mathrm{R}}^{2}=1.23 \cdot C_{\mathrm{n}}^{2} \cdot k^{7 / 6} \cdot L^{11 / 6}
$$

where $k=2 \pi / \lambda$ is the wave number and $\lambda$ is the wavelength, $L$ is the distance between the transmitter and the receiver, and $C_{n}^{2}$ is the index of refraction structure parameter and is used as a measure of turbulence strength. For horizontal path propagation, the parameter $C_{n}^{2}$ is assumed to be constant with average values of $10^{-17} \mathrm{~m}^{-2 / 3}$ to $10^{-13} \mathrm{~m}^{-2 / 3}$, for weak to strong turbulence channel respectively [12].

\subsection{Pointing Error Fading Model}

A pointing error due to buildings sway is considered and assumed to be independent from atmospheric turbulence. Furthermore, the pointing error process was modeled using the approach developed by [15] which assumes a Gaussian beam with beam waist $w_{Z}$ (radius calculated at $\mathrm{e}^{-2}$ ) and circular detection aperture of radius $a$. In this case, the fraction of collected power at a receiver due to geometric spread with radial displacement $r$ can be well approximated by [15]

$$
h_{\mathrm{p}}(r) \approx A_{\mathrm{o}} \cdot \exp \left(-\frac{2 r^{2}}{w_{\mathrm{z}_{\mathrm{eq}}}^{2}}\right)
$$

where $v=\sqrt{(\pi / 2)} \cdot a / w_{z}, A_{o}=[\operatorname{erf}(v)]^{2}$ is the fraction of collected power at $r=0$, while $\operatorname{erf}(\cdot)$ is the error function and $w_{\mathrm{z}_{\mathrm{eq}}}^{2}=\frac{w_{z}^{2} \cdot \operatorname{erf}(v) \cdot \sqrt{\pi}}{2 v \cdot \exp \left(-v^{2}\right)}$. It was further assumed that the radial displacement $r$ at the receiver is Rayleigh distributed expressed as

$$
f_{\mathrm{r}}(r)=\frac{r}{\sigma_{\mathrm{s}}^{2}} \cdot \exp \left(-\frac{r^{2}}{2 \sigma_{\mathrm{s}}^{2}}\right), \quad r>0
$$

where $\sigma_{\mathrm{s}}^{2}$ is the jitter variance at the receiver. Using (11) and (12), the pdf of the pointing error $h_{\mathrm{p}}$ can be expressed as [15]

$$
f_{\mathrm{h}_{\mathrm{p}}}\left(h_{p}\right)=\frac{\gamma^{2}}{A_{\mathrm{o}}^{\gamma^{2}}} \cdot h_{\mathrm{p}}^{\gamma^{2}-1}, 0 \leq h_{\mathrm{p}} \leq A_{\mathrm{o}}
$$

The parameter $\gamma=w_{\text {zeq }} / 2 \sigma_{\mathrm{s}}$ is the ratio between the equi- valent beam radius at the receiver and the pointing error displacement standard deviation at the receiver.

\subsection{Channel Statistical Model}

The channel state $h$ as depicted by (2), is random and was assumed to be the product of the three independent variables, $h=h_{\mathrm{a}} \cdot h_{\mathrm{o}} \cdot h_{\mathrm{p}}$, the pdf of the channel state $h$ can be derived by using

$$
f_{\mathrm{h}}(h)=\int f_{\mathrm{h} \mid \mathrm{h}_{\mathrm{a}}}\left(h \mid h_{\mathrm{a}}\right) f_{\mathrm{h}_{\mathrm{a}}}\left(h_{\mathrm{a}}\right) \mathrm{d} h_{\mathrm{a}}
$$

where the conditional probability $f_{\mathrm{h} \mid \mathrm{h}_{\mathrm{a}}}\left(h \mid h_{\mathrm{a}}\right) \mathrm{s}$ given by [15]

$$
\begin{aligned}
f_{\mathrm{h} \mid \mathrm{h}_{\mathrm{a}}}\left(h \mid h_{\mathrm{a}}\right) & =\frac{1}{h_{\mathrm{a}} h_{\mathrm{o}}} f_{\mathrm{h}_{\mathrm{p}}}\left(\frac{h}{h_{\mathrm{a}} h_{\mathrm{o}}}\right)=\frac{\gamma^{2}}{A_{\mathrm{o}}^{\gamma^{2}} h_{\mathrm{a}} h_{\mathrm{o}}}\left(\frac{h}{h_{\mathrm{a}} h_{\mathrm{o}}}\right)^{\gamma^{2}-1}, \\
0 & \leq h \leq A_{\mathrm{o}} h_{\mathrm{a}} h_{\mathrm{o}}
\end{aligned}
$$

By substituting (6) and (15) into (14), an expression for the pdf of the channel state is

$$
\begin{aligned}
f_{\mathrm{h}}(h)= & \frac{2 \gamma^{2}(\alpha \beta)^{0.5(\alpha+\beta)}}{\left(A_{\mathrm{o}} h_{\mathrm{o}}\right)^{\gamma^{2}} \Gamma(\alpha) \Gamma(\beta)} \cdot h^{\gamma^{2}-1} . \\
& \int_{h / A_{\mathrm{o}} h_{\mathrm{o}}}^{\infty} h_{\mathrm{a}}^{0.5(\alpha+\beta)-1-\gamma^{2}} \cdot \mathrm{K}_{\alpha-\beta}\left(2 \sqrt{\alpha \beta h_{\mathrm{a}}}\right) \mathrm{d} h_{\mathrm{a}} .
\end{aligned}
$$

The above expression was later simplified by [13] in terms of the Meijer's G-function and has the following closed form expression

$$
\begin{aligned}
& f_{\mathrm{h}}(h)= \frac{\gamma^{2} \alpha \beta}{A_{\mathrm{o}} h_{\mathrm{o}} \Gamma(\alpha) \Gamma(\beta)}\left(\frac{\alpha \beta h}{A_{\mathrm{o}} h_{\mathrm{o}}}\right)^{0.5(\alpha+\beta)-1} . \\
& G_{1,3}^{3,0}\left[\frac{\alpha \beta}{A_{\mathrm{o}} h_{\mathrm{o}}} h \mid \begin{array}{c}
1-\frac{\alpha+\beta}{2}+\gamma^{2} \\
-\frac{\alpha+\beta}{2}+\gamma^{2}, \frac{\alpha-\beta}{2}, \frac{\beta-\alpha}{2}
\end{array}\right] .
\end{aligned}
$$

\subsection{Average Signal-to-Noise Ratio}

The average signal-to-noise ratio $\bar{u}$ can be derived using (5) as

$$
\bar{u}=E[S N R(h)]=\frac{2 \cdot P_{\mathrm{t}}^{2} \cdot R^{2}}{\sigma_{\mathrm{n}}^{2}} \cdot E\left[h^{2}\right]
$$

where $E\left[h^{2}\right]$ is the second moment of the channel state $h$. Thus, the average received SNR can be expressed as

$$
\bar{u}=\frac{2 \cdot P_{\mathrm{t}}^{2} \cdot R^{2}}{\sigma_{\mathrm{n}}^{2}} \cdot \int_{0}^{\infty} h^{2} \cdot f_{\mathrm{h}}(h) \mathrm{d} h .
$$

By using $\mathrm{d}(17,07.34 .21 .009 .01)$ by [17], and using the identity $\Gamma(a+b)=(b+a-1)$ !, the average SNR has the form

$$
\bar{u}=\frac{2 \cdot P_{\mathrm{t}}^{2} \cdot R^{2}}{\sigma_{\mathrm{n}}^{2}} \cdot \frac{(1+\alpha)(1+\beta) \gamma^{2} A_{\mathrm{o}}^{2} \cdot h_{\mathrm{o}}^{2}}{\alpha \beta\left(2+\gamma^{2}\right)}
$$


where the notation $(N)$ ! means the factorial of $N$ and $\Gamma(\cdot)$ is the gamma function.

\section{System Metrics Derivation and Optimization}

For the analysis, an IM/DD FSO system using OOk modulation is considered that is influenced by the combined effect of turbulence channels, pointing error and weather conditions. In addition, the background noise is assumed to be zero-mean additive white Gaussian noise with variance $\sigma_{\mathrm{n}}^{2}$.

\subsection{Average Bit Error Rate}

For the system under study, the condition on $h$ probability of error is given by

$$
P(e \mid h)=\mathrm{Q}\left(\frac{\sqrt{2} p_{\mathrm{t}} \cdot h}{\sigma_{\mathrm{n}}}\right)
$$

where $\mathrm{Q}(x)$ is the Gaussian Q-function, $Q(x)=\frac{1}{\sqrt{2 \pi}} \int_{x}^{\infty} \exp \left(-\frac{y^{2}}{2}\right) d y$, and can represented by the complimentary error function $\operatorname{erfc}(x)=2 \mathrm{Q}(\sqrt{2} x)$. For equiprobable transmission; $p(0)=p(1)=0.50$, the condition on the channel state $h$ probability of error can be expressed as

$$
p(e \mid h)=p(e \mid 0, h)=p(e \mid 1, h)=\frac{1}{2} \operatorname{erfc}\left(\frac{p_{\mathrm{t}} \cdot h}{\sigma_{\mathrm{n}}}\right) .
$$

The average BER can be found by averaging the conditional probability $p(e \mid h)$ over the channel distribution $f_{\mathrm{h}}(h)$ as

$$
P(e)=\int_{0}^{\infty} f_{\mathrm{h}}(h) \cdot P(e \mid h) \mathrm{d} h .
$$

Using (06.27.26.0006.01) given by [17], the erfc $(x)$ in (20) can be represented in terms of the Meijer's $G$ function as

$$
\operatorname{erfc}\left(\frac{p_{\mathrm{t}} \cdot h}{\sigma_{\mathrm{n}}}\right)=\frac{1}{\sqrt{\pi}} G_{1,2}^{2,0}\left[\left(\frac{p_{\mathrm{t}} \cdot h}{\sigma_{\mathrm{n}}}\right)^{2} \mid \begin{array}{r}
1 \\
0, \frac{1}{2}
\end{array}\right] .
$$

By using (21) developed by [18], a closed form solution for the BER can be obtained as

$$
\begin{aligned}
& P(e)=\frac{2^{\alpha+\beta-3} \gamma^{2}}{\Gamma(\alpha) \Gamma(\beta) \sqrt{\pi^{3}}} \cdot \\
& G_{7,4}^{2,6}\left[\begin{array}{c}
\frac{8 \bar{u}\left(2+\gamma^{2}\right)}{\alpha \beta \gamma^{2} R(1+\alpha)(1+\beta)} \mid \frac{1-\gamma^{2}}{2}, \frac{2-\gamma^{2}}{2}, \frac{1-\alpha}{2}, \frac{2-\alpha}{2}, \frac{1-\beta}{2}, \frac{2-\beta}{2}, 1 \\
0, \frac{1}{2},-\frac{\gamma^{2}}{2}, \frac{1-\gamma^{2}}{2}
\end{array}\right]
\end{aligned}
$$

Using (7.34.03.0001.01) and (7.34.16.0002.01) developed by [17], $P(e)$ can be simplified to have the form

$$
\begin{aligned}
p(e)= & \frac{2^{\alpha+\beta-3} \gamma^{2}}{\Gamma(\alpha) \Gamma(\beta) \sqrt{\pi^{3}}} \cdot \\
& G_{3,6}^{5,2}\left[\frac{\alpha \beta \gamma^{2} R(1+\alpha)(1+\beta)}{8 \bar{u}\left(2+\gamma^{2}\right)} \mid \begin{array}{c}
1, \frac{1}{2}, 1+\frac{\gamma^{2}}{2} \\
\left.\frac{\gamma^{2}}{2}, \frac{1+\alpha}{2}, \frac{\alpha}{2}, \frac{1+\beta}{2}, \frac{\beta}{2}, 0\right]
\end{array}\right]
\end{aligned}
$$

Alternatively, using (20), $p(e)$ can be expressed in terms of transmitted power

$$
\begin{aligned}
P(e)= & \frac{2^{\alpha+\beta-3} \gamma^{2}}{\Gamma(\alpha) \Gamma(\beta) \sqrt{\pi^{3}}} . \\
& G_{3,6}^{5,2}\left[\left(\frac{\alpha \cdot \beta}{4 A_{\mathrm{o}} h_{\mathrm{o}}\left(p_{\mathrm{t}} / \sigma_{\mathrm{n}}\right)}\right)^{2} \mid \frac{\gamma^{2}}{2}, \frac{1+\alpha}{2}, \frac{\alpha}{2}, \frac{1+\frac{\gamma^{2}}{2}}{2}, \frac{\beta}{2}, 0\right] .
\end{aligned}
$$

For a given average received $\mathrm{SNR}, \bar{u}$, in the cases of weak to strong turbulence conditions and normalized jitter values $\sigma_{\mathrm{sa}} \geq 2$, the optimal normalized beamwidth, $\left(w_{\mathrm{za}}\right)_{\mathrm{op}}$, can be determined as to minimize the BER. Since the BER is a decreasing function of the beamwidth, the expression given in (26) cannot be minimized directly as this leads theoretically to an infinite beamwidth value. However, $p(e)$ maintains approximately a constant slope starting at the point of the optimal beamwidth, therefore the tail of $p(e)$ can be approximated by a straight line with slope approaches zero. Therefore, a penalty factor is added to the cost function obtained from (26), which locates the point on $P(e)$ curve where $p(e)$ is minimum and has a minimum slope, this point is the optimal normalized beamwidth that minimizes BER. The overall cost to optimize BER can be written as

$$
F\left(P(e), w_{\mathrm{za}}, \bar{u}\right)=P(e)+P(e) w_{\mathrm{za}}
$$

where $w_{\mathrm{za}}$ in (28) is related to $\gamma$ in (26) as the expression in (11). The optimization in (28) can be performed using deterministic search algorithms such as Nelder-Mead simplex [24], or random methods such as Swarm optimization. For instance, in Nelder-Mead algorithm [24], an iterative simplex is formed in each iteration where the solution is represented by the coordinates of each vertex. The vertices are updated according to their fitness values, in our case the objective given in (28). During the optimization process, the algorithm uses reflection and scaling operations to form a better simplex until it terminates with the optimal solution, $\left(w_{\text {za }}\right)_{\text {op. }}$.

Similarly, the minimum $p(e)$ can be obtained in terms of transmitted power for weak to strong channel turbulence and different weather conditions and propagation path 
lengths. In this case, the optimal values for the normalized beamwidth and the transmitted power are determined as to minimize BER. The optimization can be performed by minimizing the expression of $P(e)$ given in (27) directly using Nelder-Mead technique. It should be noted that in (27), $P(e)$ has a unique minimum with respect to $w_{\mathrm{za}}, \sigma_{\mathrm{sa}}$ and $p_{\mathrm{t}}$.

\subsection{Probability of Outage}

For a given source rate $R_{\mathrm{o}}$ symbols/sec, outage event occurs when the channel capacity $C$ cannot support $R_{\mathrm{o}}$, $C>R_{\text {o, }}$, when this event takes place, the receiver cannot decode the transmitted symbols correctly with an arbitrarily small error. Equivalently, the outage event can also take place when the instantaneous SNR falls below a minimum received SNR value, $u_{\min }$. The probability of this outage event is termed outage probability $P_{\text {out }}$ and is given by

$$
P_{\text {out }}=p_{\mathrm{r}}\left(u<u_{\min }\right) \text {. }
$$

By using (4), the above probability can be written as

$$
P_{\text {out }}=p_{\mathrm{r}}\left(h<\sqrt{u_{\min } \sigma_{\mathrm{n}}^{2} / 2 P_{\mathrm{t}}^{2} \cdot R^{2}}\right)
$$

where the channel state $h$ has the distribution as in (17). Thus, the outage probability is the cumulative distribution of $h$

$$
P_{\text {out }}=F_{\mathrm{h}}(h)=\int_{0}^{h_{\min }} p_{\mathrm{h}}(h) \mathrm{d} h
$$

where $h_{\text {min }}=\sqrt{u_{\min } \sigma_{\mathrm{n}}^{2} / 2 P_{\mathrm{t}}^{2} \cdot R^{2}}$. By using (26) given by [16], a closed form solution is found for $P_{\text {out }}$ and has the form

$$
\begin{aligned}
& p_{\text {out }}=\frac{\gamma^{2}}{\Gamma(\alpha) \Gamma(\beta)}\left(\frac{\alpha \beta}{A_{\mathrm{o}} h_{\mathrm{o}}}\right)^{(\alpha+\beta) / 2} . \\
& G_{2,4}^{3,1}\left[\begin{array}{l|c}
\frac{\alpha \beta}{A_{\mathrm{o}} h_{\mathrm{o}}} h_{\min } & 1-\frac{\alpha+\beta}{2}, 1-\frac{\alpha+\beta}{2}+\gamma^{2} \\
-\frac{\alpha+\beta}{2}+\gamma^{2},-\frac{\alpha-\beta}{2}, \frac{\beta-\alpha}{2},-\frac{\alpha+\beta}{2}
\end{array}\right] .
\end{aligned}
$$

By using (7.34.16.0001.1) by [17], $p_{\text {out }}$ can be simplified as

$$
p_{\text {out }}=\frac{\gamma^{2}}{\Gamma(\alpha) \Gamma(\beta)} G_{2,4}^{3,1}\left[\frac{\alpha \beta}{A_{\mathrm{o}} h_{\mathrm{o}}} h_{\min } \mid \begin{array}{c}
1,1+\gamma^{2} \\
\gamma^{2}, \alpha, \beta, 0
\end{array}\right] .
$$

Alternatively, $p_{\text {out }}$ can be expressed in terms of average SNR

$$
\begin{aligned}
& p_{\text {out }}=\frac{\gamma^{2}}{\Gamma(\alpha) \Gamma(\beta)} \cdot \\
& G_{2,4}^{3,1}\left[\gamma \sqrt{\frac{u_{\min }(1+\alpha)(1+\beta) \cdot \alpha \beta}{\bar{u}\left(2+\gamma^{2}\right)}} \mid \begin{array}{c}
1,1+\gamma^{2} \\
\gamma^{2}, \alpha, \beta, 0
\end{array}\right] .
\end{aligned}
$$

The probability of outage for weak to strong turbulence conditions, and different weather conditions and normalized jitter values $\sigma_{\mathrm{sa}} \geq 2$, can also be optimized with respect to the average $\mathrm{SNR}, \bar{u}$, and transmitted power and the beamwidth using the same procedures presented in Sec. 3.1.

During the optimization process in both cases, $p(e)$ and $P_{\text {out }}$, it should be noted that the value of $\left(w_{z a}\right)_{\text {op }}$ for $\sigma_{\mathrm{sa}}=2$ achieves the minimum BER and outage probability. Therefore, the optimized normalized beamwidth, obtained for a specific average SNR can be taken as a reference for both system metrics for jitter values, $\sigma_{\mathrm{sa}}>2$. In this case, the obtained results for $p(e)$ and $P_{\text {out }}$ are higher when referenced to the optimal beamwidth in the case of $\sigma_{\mathrm{sa}}=2$. In all cases, for any given values of $\sigma_{\mathrm{sa}}, p_{\mathrm{t}}$ and $\bar{u}$, the optimum normalized beamwidth, $\left(w_{\mathrm{za}}\right)_{\mathrm{op}}$, was found by varying $w_{\mathrm{za}}$ between 2 and 25 .

\section{Numerical Results}

Table 1 summarizes the different weather conditions and system parameters used in this section assuming a noise standard deviation $\sigma_{\mathrm{n}}=10^{-7} \mathrm{~A} / \mathrm{Hz}$. In addition, the following Rytov variance values: $\sigma_{\mathrm{R}}{ }^{2}=0.50,1,0,5.0$ were used for weak to strong turbulence channel severity. In generating the optimized results, the optimized normalized beamwidth $\left(w_{\text {za }}\right)_{\text {op }}$ was found for each case of $\left(\sigma_{\mathrm{sa}}, \bar{u}, w_{\mathrm{za}}\right)$ by varying the values of $w_{z a}$ in (28) between 2 and 25 .

Using (26) and (28), Figure 1 depicts minimum BER results in terms of received average SNR for weak to strong turbulence conditions and normalized jitter values $\sigma_{\mathrm{sa}}=2,3,4$. It can be concluded from the figure, that, for any given Rytov variance and normalized jitter variance values, as the average SNR increases, optimum normalized beamwidth values increase to further minimize BER performance, which agrees well with the findings by [13]. In addition, for any given jitter variance and average SNR, as turbulence severity gets stronger, the value of optimum beamwidth required to achieve minimum BER results decreases.

Using (27), Figure 2 shows minimum BER performance in terms of transmitted power for weak to strong channel turbulence and light haze weather condition. The

\begin{tabular}{|l|c|c|c|c|}
\hline \multicolumn{5}{|c|}{$\lambda=1550 \mathrm{~nm}, R=1.0$} \\
\hline $\begin{array}{l}\text { Weather } \\
\text { condition }\end{array}$ & $\begin{array}{c}\text { Visibility } \\
\text { range }(\mathrm{km})\end{array}$ & $\begin{array}{c}\text { Range } \\
(\mathrm{km})\end{array}$ & $h_{\mathrm{o}}(L)$ & $\begin{array}{c}\text { Attenuation } \\
\mathrm{dB} / \mathrm{km}\end{array}$ \\
\hline Clear & 10.0 & 1 & 0.9033 & \multirow{2}{*}{0.4416} \\
\cline { 3 - 4 } & 4 & 4 & 0.6658 & \\
\hline Light haze & 2.0 & 2 & 0.4738 & \\
\cline { 3 - 4 } Dense haze & $0.0892(.5 \mathrm{~km})$ & 1.5 & 0.4021 & \multirow{2}{*}{3.956} \\
\cline { 3 - 4 } & $0.0266(.75 \mathrm{~km})$ & 2 & 0.1617 & \\
\hline \multirow{2}{*}{ Fog } & \multirow{2}{*}{0.50} & 0.50 & 0.0892 & \multirow{2}{*}{20.992} \\
\cline { 3 - 4 } & & 0.75 & 0.0266 & \\
\hline
\end{tabular}

Tab. 1. System and weather condition parameters. 


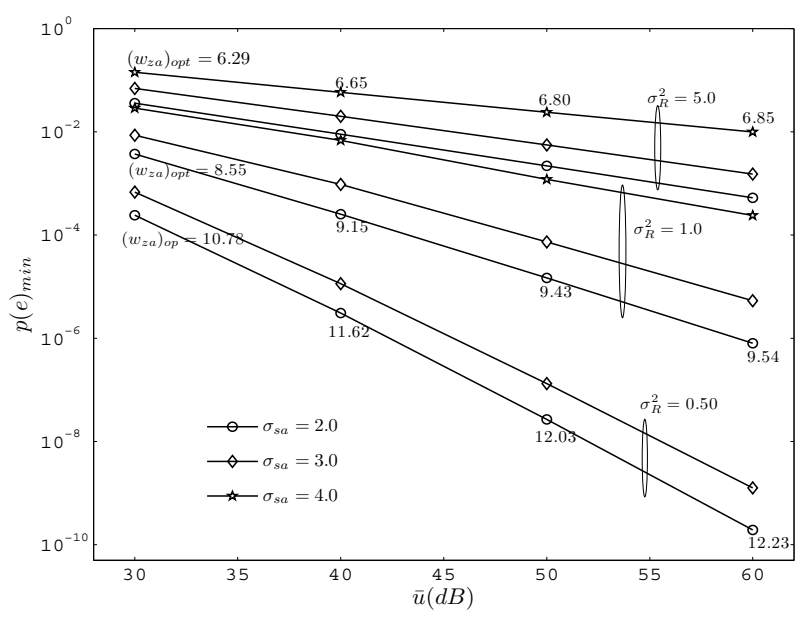

Fig. 1. Minimum BER results for different normalized jitter variances vs. average received SNR, $\bar{u}$.

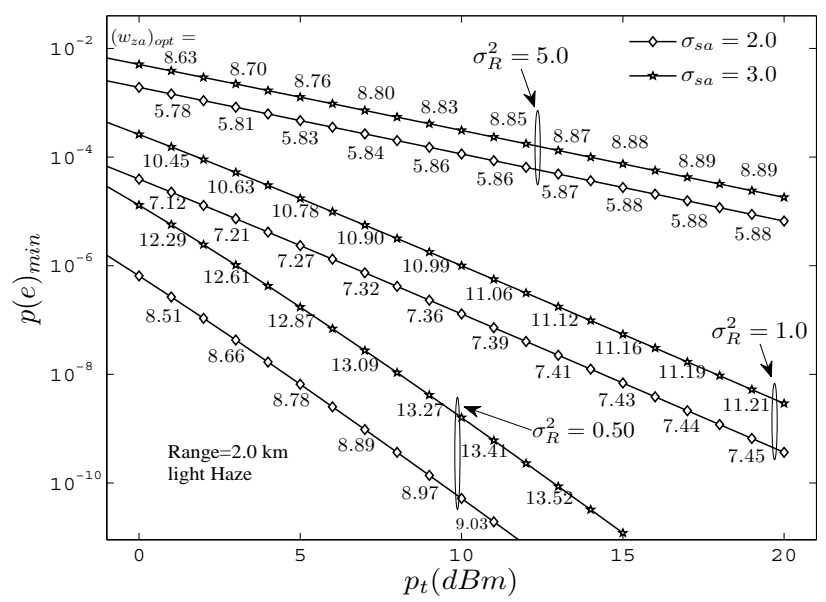

Fig. 2. Minimum BER results for weak to strong turbulence and light haze vs. transmitted power.

results are computed for jitter variance values $\sigma_{\mathrm{sa}}=2,3$, and propagation distance $L=2 \mathrm{~km}$. For any given turbulence condition and normalized jitter variance, one can achieve possible minimum BER by adjusting the system transmitted power $p_{\mathrm{t}}$ and normalized beamwidth. For example, for $\sigma_{\mathrm{R}}{ }^{2}=1.0$ and $\sigma_{\mathrm{sa}}=2.0$, at $p_{\mathrm{t}}=13.0 \mathrm{dBm}$, a minimum BER value $p(e)=2.20 \times 10^{-8}$, can be achieved by adjusting the normalized beamwidth value $\left(w_{z a}\right)_{o p}=7.41$. Figure 3 depicts BER results for different weather conditions and propagation distances. The results are computed for $\sigma_{\mathrm{R}}^{2}=2.0$ and $\sigma_{\mathrm{sa}}=3.0$. It can be observed from the figure how the BER performance is degraded due to the visibility range of the different weather conditions. As expected, the worst BER performance occurs for light fog where the visibility range is limited to $0.50 \mathrm{~km}$. It is interesting to note, that, for any given weather conditions and propagation distance, $\left(w_{\text {za }}\right)_{\text {op }}$ value that achieves minimum $p(e)$ is almost the same for $p_{\mathrm{t}} \geq 17 \mathrm{dBm}$.

Figures 4 and 5 depict probability of outage for weak to strong turbulence conditions and normalized jitter values $\sigma_{\mathrm{sa}}=2,3,4$. It can be seen from the figures, as the value of

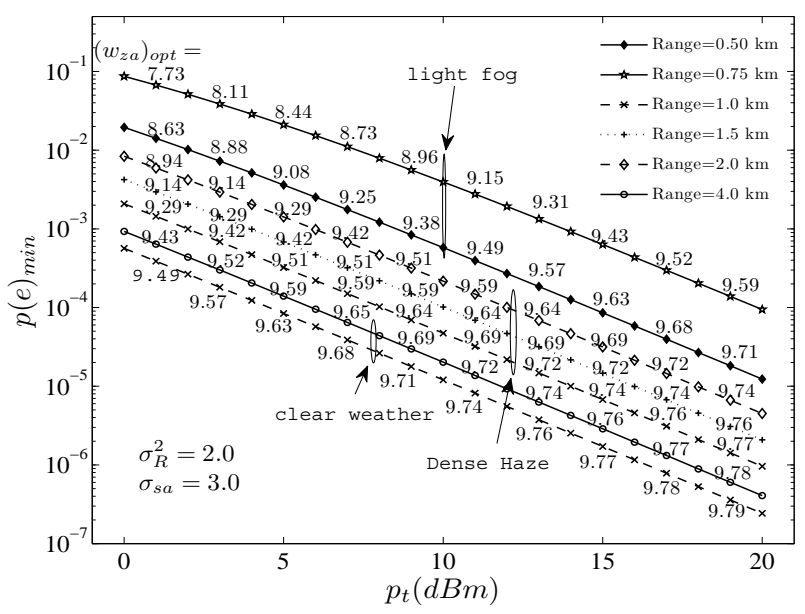

Fig. 3. Minimum BER results for different weather conditions and distances vs. transmitted power.

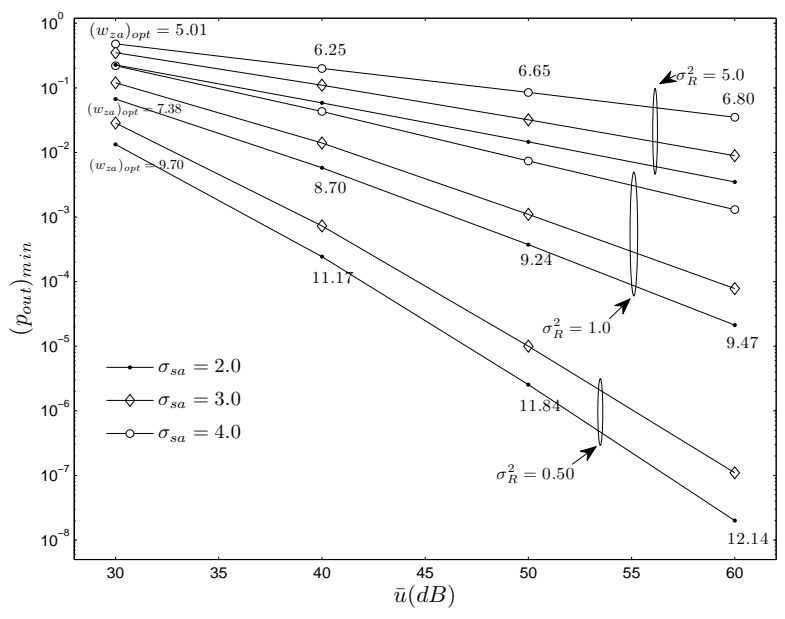

Fig. 4. Minimum outage probability for different normalized jitter variances vs. average received SNR, $\bar{u}$.

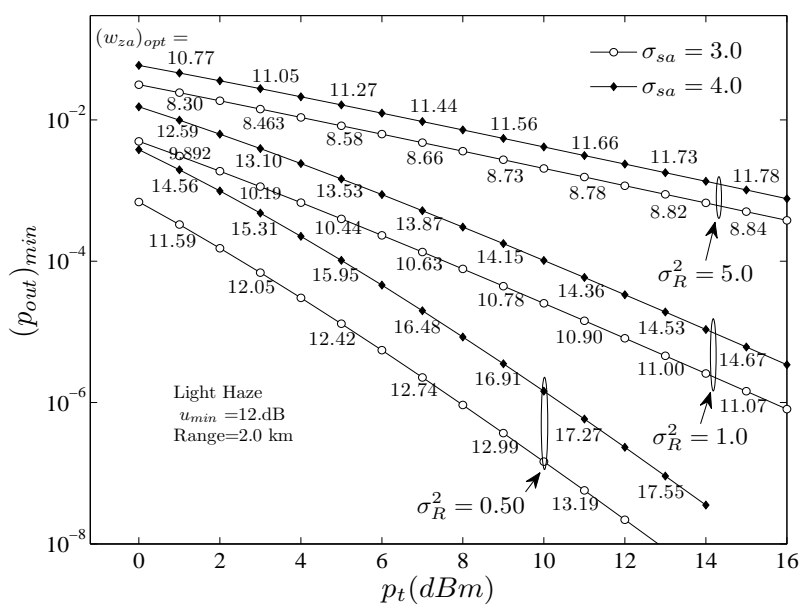

Fig. 5. Minimum outage probability for weak to strong turbulence and light haze vs. transmitted power.

average SNR or transmitted power increase, optimum normalized beamwidth values needed to achieve minimum outage probability increase, too. In addition, as the Rytov 


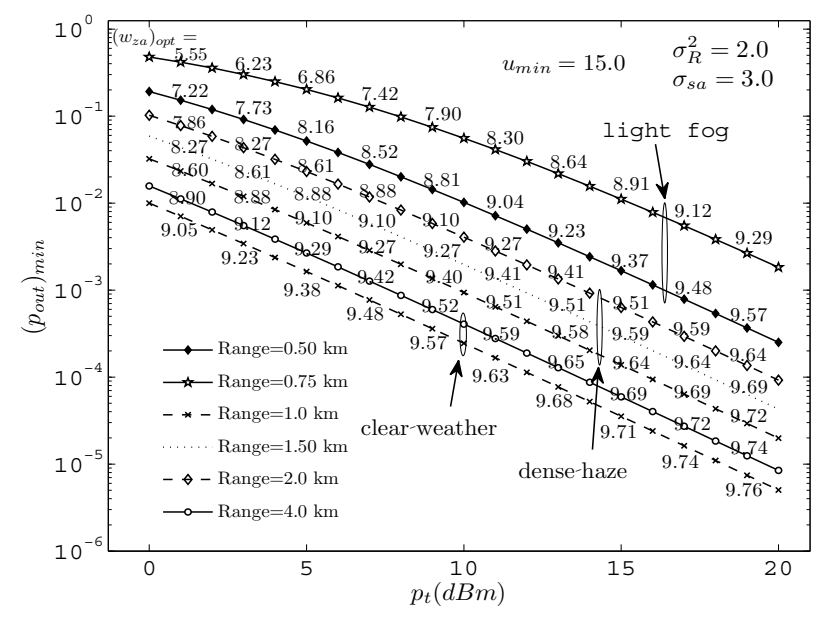

Fig. 6. Minimum outage probability for different weather conditions and distances vs. transmitted power.

variance moves from weak to strong turbulence strength, minimum outage probability is achieved by decreasing the laser beamwidth.

Probability of outage results for different weather conditions and propagation distances are shown in Fig. 6, using $\sigma_{\mathrm{R}}^{2}=2.0$ and $\sigma_{\mathrm{sa}}=3$. The figure exhibits the same behavior as Fig. 3, where fog weather condition highly degrades outage performance. Finally, by increasing the link operational range, minimum outage probability results are attained by adjusting the laser beamwidth to lesser values.

\section{Conclusion}

In this paper, an FSO system with IM/DD and on-off signaling that operates over atmospheric slowly fading gamma-gamma turbulence channels was analyzed. Novel BER and outage probability closed form expressions are derived taking into account the combined effects of atmospheric turbulence, path loss due to weather conditions and pointing error. Optimized normalized beamwidth values that achieve minimum BER and outage probability were found for different SNR and transmitted power values. For each case of SNR and transmitted power, the optimized beamwidths were found by taking into account the combined effects of turbulence strengths, normalized jitter variance, weather condition and the length of the propagation path.

As the average SNR or the transmitted power value increases, minimum BER and outage probability results are attained by increasing the laser beamwidth regardless of the jitter variance, turbulence strength and weather conditions. It was also found that increasing the path loss or moving from weak to strong turbulence channel conditions, minimum BER and outage probability results can be achieved by decreasing the laser beamwidth. Finally, for higher SNR and transmitted power values, optimized beamwidth values that achieve minimum BER and outage probability results are practically the same values.

\section{References}

[1] KIM, I., CHAFFEE, T., FLEISHAUER, R., et al. Advances in Communications: New FSO provides reliable $10 \mathrm{Gbit} / \mathrm{s}$ and beyond backhaul connections. Laser Focus World, 2013, p. 1-8.

[2] http://www.attochron.com/

[3] KIM, I., MCARTHUR, B., KOREVAAR, E. Comparison of laser propagation at $785 \mathrm{~nm}$ and $1550 \mathrm{~nm}$ in for fog and haze for optical wireless communications. SPIE Proceedings, 2001, vol. 4214, p. 26-37. DOI: $10.1117 / 12.417512$

[4] HASAN, O. Performance of heterodyne differential phase-shift keying system over double Weibull free-space optical channel. Journal of Modern Optics, 2015, vol. 62, no. 11, p. 869-876. DOI: 10.1080/09500340.2015.1027311

[5] TRUNG, H., TUAN, D., PHAM, A. Pointing error effects on performance of free-space optical communication system using SC-QAM signals over atmospheric turbulence channels. $A E U$ International Journal of Electronics and Communications, 2014, vol. 68, no. 9, p. 869-876. DOI: 10.1016/j.aeue.2014.04.008

[6] VU, B., DANG, N., THANG, T., PHAM, A. Bit error rate analysis of rectangular QAM/FSO systems using an APD receiver over atmospheric turbulence channels. Journal of Optical Communications and Networking. 2013, vol. 5, no 5, p. 437-446. DOI: 10.1364/JOCN.5.000437

[7] MORRA, A., KHAllaF, H., SHALABY, H., KAWASAK, Z. Performance analysis of both shot- and thermal-noise limited multipulse PPM receivers in gamma-gamma atmospheric channels. Journal of Lightwave Technology, 2013, vol. 31, no. 19, p. 3142-3150. DOI: 10.1109/JLT.2013.2278692

[8] NISTAZAKES, H., TSIGOPOUlOS, A., HANIAS, M., et al. Estimation of outage capacity for free space optical links over I-K and $\mathrm{K}$ turbulence channels. Radio Engineering, 2011, vol. 20, no. 11, p. $493-498$.

[9] CHATZIDIAMANTIS, N., SANDALIDIS, H., KARAGIANNIDIS, G., et al. New results on turbulence modeling for free-space optical systems. In Proceedings of the 17th IEEE International Conference on Telecommunications. Doha (Qatar), April, 2010. DOI: 10.1109/ICTEL.2010.5478872

[10] POPOOLA, W., GHASSEMLOOY, Z., AHMADY, V. Performance of subcarrier modulated free-space optical communication link in negative exponential atmospheric turbulence environment. International Journal of Autonomous and Adaptive Communication Systems, 2008, vol. 1, no. 3, p. 342-355. DOI: 10.1504/IJAACS.2008.019809

[11] AL-HABASH, C., ANDREWS, L., PHILIPS, R. Mathematical model for irradiance probability density function of a laser beam propagating through turbulent media. Optical Engineering, 2001, vol. 40 , no. 8 , p. $1554-1563$. DOI: $10.1117 / 1.1386641$

[12] NISTAZAKIS, H., KARAGIANI, E., TSIGOPOULOS, A., et al. Average capacity of optical wireless communication systems over atmospheric turbulence channels. Journal of Lightwave Technology, 2009, vol. 27, no. 8, p. 974-979. DOI: 10.1109/JLT.2008.2005039

[13] SANDALIDIS, H., TSIFTSIS, A., KARAGIANNIS, G. Optical wireless communications with heterodyne detection over turbulence channels with pointing errors. Journal of Lightwave Technology, 2009, vol. 27, no. 20, p. 4440-4445. DOI: 10.1109/JLT.2009.2024169

[14] SANDALIDIS, H., TSIFTSIS, T. Outage probability and ergodic capacity of free-space optical links over strong turbulence. Electronics Letters, 2008, vol. 44, no. 1, p. 46-47. DOI: 10.1049/el:20082495

[15] FARID, A., HRANILOVIC, S. Outage capacity optimization for free-space optical links with pointing errors. Journal of Lightwave Technology. 2007, vol. 25, no. 7, p. 1702-1710. DOI: 10.1109/JLT.2007.899174 
[16] SAGIAS, N., KARAGIANNIDIS, G., MATHIOPOULOS, P., et al. On the performance analysis of equal-gain diversity receiver over generalized Gamma fading channels. IEEE Transactions on Wireless Communications, 2006, vol. 5, no. 10, p. 2967-2973. DOI: 10.1109/TWC.2006.05301.

[17] Wolfram function site, http://functions.wolfram.com.

[18] ADAMCHIK, V., MARIACHEV, O. The algorithm for calculating integrals of hypergeometric type and its realization in reduce system. In Proceedings of the International Symposium on Symbolic and Algebraic Computation (ISSAC'90). Tokyo (Japan), 1990, p. 212-224. DOI: 10.1145/96877.96930

[19] TSIFTSIS, T., SANDALIDIS, H., KARAGIANNIDIS, G., et al. Optical wireless links with diversity over strong atmospheric turbulence channels. IEEE Transactions on Wireless Communications, 2009, vol. 8, no. 2, p. 951-957. DOI: 10.1109/TWC.2009.071318

[20] KIASALEH, K. Performance of coherent DPSK free-space communication systems in $\mathrm{K}$-distributed turbulence. IEEE Transactions on Communications, 2006, vol. 54, no. 4, p. 604-607. DOI: 10.1109/TCOMM.2006.873067

[21] PHILLIPS, R., ANDREWS, L. Measured statistics of laser light scattering in atmospheric turbulence. Journal of the Optical Society of America, 1981, vol. 71, no. 12, p. 1440-1445. DOI: 10.1364/JOSA.71.001440

[22] FLATTE, S., BRACHER, C., WANG, G. Probability-density functions of irradiance for waves in atmospheric turbulence calculated by numerical simulations. Journal of the Optical Society of America A, 1994, vol. 11, no. 7, p. 2080-2092. DOI: 10.1364/JOSAA.11.002080

[23] CHOUDHURY, S., GIBSON, J. D. Ergodic Capacity, Outage Capacity, and Information Transmission over Rayleigh Fading Channels. Dept. of Electrical and Computer Engineering, University of California, Santa Barbara. 5 pages. [online] Cited 2015-04-10. Available at:

http://vivonets.ece.ucsb.edu/ChoudhuryGibson_ITW07.pdf.

\section{About the Authors ...}

Omar HASAN received his B.Sc degree in Electrical
Engineering in 1987, M.Sc and Ph.D degrees in Telecommunication in 1990 and 1996, respectively, all from New Mexico State University, USA. He joined Princess Sumaya University for Technology in 1997 where he served as a chairman for the communications engineering department in the period 2005-2010 and the dean of student affairs. He worked in different projects at physical science laboratory, Las Cruces, New Mexico, USA, in the period 1991-1992. In summer 2000, he worked at Global Cardiac Monitors, research and development division in the area of Turbo coding, Webster, Texas, USA. He worked as a system analyst at Intelligent Technology Incorporated, Telecommunications division, Las Cruces, New Mexico, USA 1989-1992. He worked as a consultant for Global Cardiac Monitors LLC, Houston, Texas, USA, 2001, 2004, and 2012-current. He worked as a research assistant at the manual Lujan, Jr. center for Space and Telemetering and Telecommunications, Simulation and Tracking system division, New Mexico State University, Las Cruces, New Mexico, USA, 1996. His current research interest includes wireless optical communications and low power coding schemes. Dr. Hasan received research funding from the Higher Council for Science and Technology, development and utilization of DC and AC inverters in solar cells energy, 1999, Amman, Jordan.

Mohamed TAHA is with the department of communication engineering, Princess Sumaya University for Technology. He obtained his BSc. degree with distinction in Electronics Engineering from Princess Sumaya University for Technology in 2000. He received his M.Sc degree in Communication Engineering from the University of Jordan in 2007. He serves as a reviewer for the IEEE Transactions in Wireless Communication and several international conferences. His research interest includes signal processing in wireless communication, statistical signal processing and soft computing. 